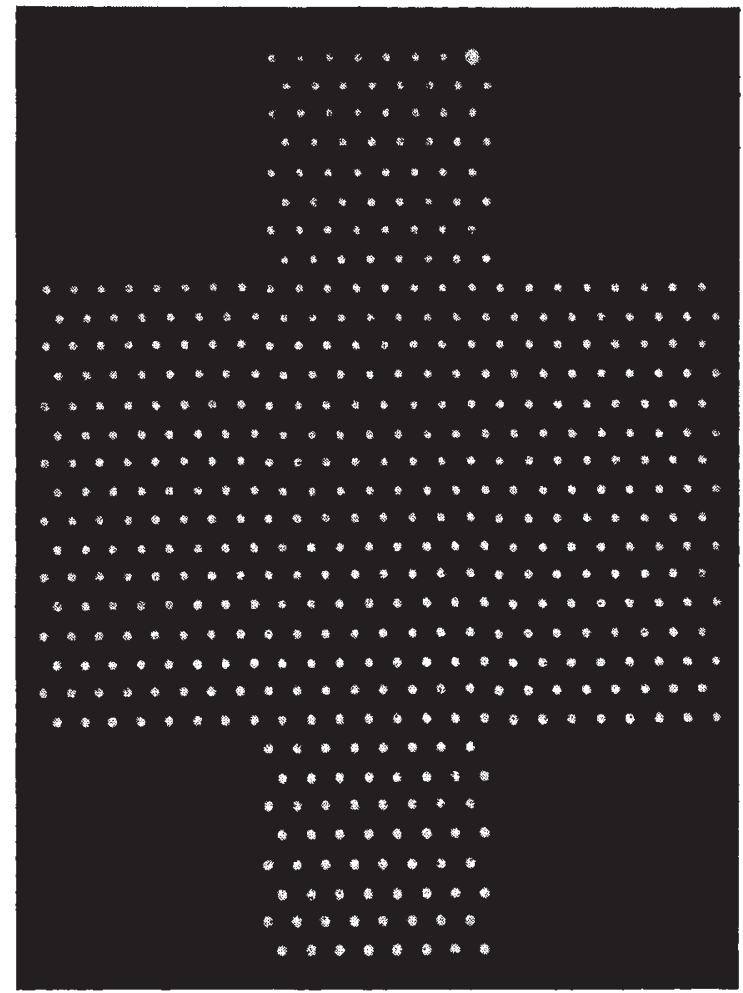

Crown copyright reserved

with 256 digits using the above techniques. This was used in conjunction with an experimental portion of the computer.

On further consideration, however, the defocusfocus system appeared to have many advantages over the modulation system. These are: (1) the refilling process by means of the focused central dot is much more efficient than that using a displaced dot, being effected by electrons emitted in all directions, instead of by those emitted only in a particular sector; as a result, the net beam current may be reduced with a consequent reduction of interaction; (2) since the defocused inspection spot and the focused refilling spot are concentric, the chances that the charge barrier due to an impurity of the screen would prevent the re-establishment of the charge are very slight; (3) the charge of the digit is detected by a defocused spot, and the system is therefore less sensitive to errors of the deflexion voltage than when using a focused beam; (4) there should be an improvement in signal strength due to the increased quantity of charge in the defocused spot.

Experiments have confirmed these advantages. The duration of re-filling pulses can be reduced to 0.8 usec. compared with about 2 usec. with the double-dot system, and the total time for reading or writing a digit can be reduced to $2 \mu$ sec.

Many cathode-ray tubes have been tested to determine the effects of impurities on the screen, and all have proved completely satisfactory. One in particular, which had at least six large impurities and had been rejected for use in a dot-dash store at $1 \mathrm{kV}$., was found to be perfectly satisfactory in a defocus-focus system at $2 \mathrm{kV}$. No variation in signal strengths could be detected larger than those normally obtained due to the variation of screen surface.
Trouble previously experienced due to the micro phony of the deflexion valves completely disappeared; consequently, reliability has increased and the stability requirements of the deflexion voltages have decreased.

The increased signal strength due to the increased charge per digit of the defocus-focus system tends to compensate for the reduction of signal due to the increase of final anode voltage, and satisfactory signal/noise ratios are still obtained with $2 \mathrm{kV}$. final anode voltage. An experimental cathode-ray tube store of 512 digits using the defocus-focus system has been functioning for about six months. Each storage element may have had at least 256 regenerations adjacent to it before being itself regenerated. This is the maximum requirement, provided instructions and numbers are used alternately and are stored in different areas of the cathode-ray tube. The digits are arranged with $2 \cdot 5-\mathrm{mm}$. separation in a patterm as in the accompanying illustration.

The electrical and mechanical design of the store has been completed, and a prototype to this design tested. The twenty stores required for the computer are now in process of construction.

Experiments have also been carried out to determine whether this system is suitable for serial storage. It has been found that a square pattern of 1,024 digits ean be stored, and that any digit may have its neighbours brightened-up at least 64 times before being itself regenerated.

The cathode-ray tubes used in the above work were of type VCRX 266. This is a convenient type of 6-in. tube with low beam current and small spot diameter. All those tested were found suitable for defocus-focus storage without the need of selection.

I wish to thank members of the Research Laboratory of the General Electric Co., Ltd., for their co-operation in the development of experimental cathode-ray tubes, and the Chief Scientist of the Ministry of Supply for permission to publish this communication. [April 18.

1 Williams, F. C., and Kilburn, T., Nature, 162, 487 (1948).

${ }^{2}$ Williams, F. C., Proc. Roy. Soc., A, 185, 265 (1948).

${ }^{3}$ Williams, F. C., and Kilburn, T., Proc. Inst. Elec. Eng., 96, Part III, To. $40,81(1949)$.

${ }^{1}$ Kilburn, T., Nature, 164, 684 (1949).

\section{ABILITY OF BIRDS TO COUNT}

$T$

HE masters of 'clever' horses and dogs are often sincere in their belief that their pets really display intelligence of the highest order ; for they are quite unable to imagine that the animals can really perceive small involuntary signs and signals which tell them when the right answer has been reached and hence when they must stop barking or stamping, as the case may be. Yet it is now quite certain that that is precisely what these 'clever' animals are doing. It was not the stallion "Zarif" of Elberfeld which reproduced Descartes's "cogito ergo sum" in ill-spelt German, nor was it the dachshund "Kuno" which answered the professor's question as to what creed he had with the words "Mine is yours". These 'clever' animals only obey their master's signs which, given at the proper moment, stop the barking or stamping by which they spell out their answers in code.

In a recent article (Bull. Animal Behaviour, No. 9 ; March 1951) Prof. O. Koehler states that the achievements of certain birds with which he has experi- 
mented are examples of true learning and in no way dependent on clues, voluntary or involuntary, given by the experimenters.

During training and 'spontaneous' experiments the observer and the bird were always separated by a partition so that the experimenter was never seen by the bird; while at work he was able to watch it through the view-finder of a cine-camera fitted into the wall. Moreover, when a deterrent was required in conditioning, only one degree of punishment was used and this was always the same. It was thus impossible for the experimenter inadvertently to give signs by means of the punishing apparatus since the latter functioned along all-or-none lines.

Unlike circus animals, Prof. Koehler's birds were sometimes left entirely to themselves and the results recorded automatically so that all possibility of clues from the investigator was ruled out. The birds learned many tasks without any punishment and merely by self-conditioning. Often spontaneous experiments were made by the birds in the complete absence of any human being. The birds were often left alone with their task while the experimenter was kept far away so that even possible telepathic connexion between man and animal could be excluded. Nearly all the problems solved by the birds in spontaneous experiments were recorded automatically by film for later study.

Not even the cleverest of the birds showed inborn capacities like human beings; but they did learn unnamed numbers which were presented to them by two principal methods: (1) simultaneous presentation, and (2) successive presentation. The first of these abilities is that of being able to compare groups of units presented simultaneously side by side by seeing numbers of those units only, excluding all other clues. This problem was presented to birds in quite different ways, starting with only two groups of edible units (grains of corn, fruit, pieces of meat, etc.), the two groups differing by one unit only, and at the other extreme rising to choice according to pattern. A raven and a grey parrot were presented with five small boxes covered. with lids bearing two, three, four, five and six spots each respectively-the key being a lid with one of these numbers of spots lying on the ground in front of the boxes. Both birds learned to open only that one of the five lids which had the same number of spots as the key pattern. As a control, everything was changed in a random manner from one experiment to the next. There were fifteen positions of the five boxes and very many different positions of the key pattern; the number of units in the key pattern changed with each experiment and there were five places for the "positive" number of spots on a lid corresponding to that of the key. Moreover there were twenty-four permutations of the four negative numbers. The relative situation of the spots of one group and, with the raven only, the size and form of those spots were also changed with absolute irregularity. In the final series of experiments, for each trial a flat 'Plasticine' cake was broken into pieces of highly irregular and broken outlines, the size of a piece varying from 1 to 50 units of surface area; care was taken to make the general patterns of the positive number on the lid as unlike as possible to that of the key pattern. In an-experiment such as this it is believed that any clue foreign to the problem was excluded, and yet the raven solved the problem by choosing the positive lid according to the only item which was not changing through all the experiments, that is, the number of spots equal to that of the particular key pattern presented.

The second problem was to estimate, that is, to remember numbers of incidents following each other and thus to keep in mind numbers presented successively in time, independent of rhythm or any other clue which might be helpful. Here again many different methods were employed. At first birds were trained to eat only " $x$ " grains out of many offered without any help being given from a figure. Another task was to eat only " $x$ " peas which were rolled into a cup, one after another, at intervals ranging from 1-60 seconds. Here again there were no figures at all; the peas were delivered as if by a slot machine, one by one, the bird never seeing more than one pea in the cup. The same applied to opening lids of boxes standing in a long row until " $x$ " baits had been secured; since the baits were arranged in the boxes in twenty or more distributions from one experiment to the next, the number of lids to be opened was constantly changing. Still more remarkable was the fact that birds learned to master up to four problems of this kind at the same time. For example, a jackdaw learned to open black lids until it had secured two baits, green lids up to three, red lids up to four, and white lids until it had secured five baits. Similarly, budgerigars learned to "act upon two" when the experimenter said "dyo dyo dyo" and upon three (take only three grains from the heap) when he uttered the words "treis treis treis ...". A similar result could be obtained with a bell indicating two and a buzzer indicating three.

Since it would appear that, in this experiment, all external clues were carefully excluded, only an inner token could have been responsible for the birds ceasing action when the number was reached. The bird would appear to have made some inward marking of the units he was acting upon, and sometimes those supposed "inward marks" showed them. selves in external behaviour in the form of intention movements.

The simplest explanation offered by Prof. Koehler for this inner marking is that it may be made up of equal marks, as if human beings were to give one nod of the head for one, two for two, and so on; it is suggested that this be called "thinking unnamed numbers". On the contrary, the most prodigal assumption which could be made is that the bird did the marking by unequal or qualitatively different marks in fixed order, as if human beings think 1, 2, $3,4,5$, or, alternatively, a phrase of five different syllables. That, of course, would be named numbers, or real counting.

Apart from one case, Prof. Koehler's simplest assumption would appear to fit the facts best, there being no sign of "number intelligence" at all and no obviously progressive learning ability as in the case of children. Pigeons and budgerigars were considerably upset by changes in experimental conditions during training for a problem, whereas jackdaws grasped the transposition at once. One bird, after having learnt to open a lid with three spots and not touch a lid with four spots, was presented with three and four mealworms moving on the lids instead of spots. The bird hesitated a long time, but finally went to the three mealworms and left the four alone; instead of opening the lid it ate the mealworms from it without opening it at all.

It would thus appear that a given species of bird shows the same ability of grasping unnamed numbers whether they are presented simultaneously or suc- 
cessively; but the ability differs with the species. Thus, with pigeons it may be five or six according to experimental conditions, with jackdaws it may be six and with ravens and parrots, seven.

Similar tests of the same ability have been carried out with man. In this case the groups of figures were presented tachistoscopically by lantern slides, shown simultaneously and carefully timed so that, while they may be fully seen, the time is too short for counting to take place. It is remarkable that, when this is done with human beings, the limit of achievement is of the same order as that shown by birds. Thus, few persons reach eight and many, like pigeons, get no farther than five. Experiments are still being carried out to test the second ability ; but there is no expectation that man may achieve better results than the birds, provided named counting is excluded.

\section{LONG ASHTON RESEARCH STATION FIELD DAY}

$\mathrm{T}$

HE exhibits at the Long Ashton Research Station Field Day on July 10 included several of particular scientific interest. In the Pomology Section, Dr. L. C. Luckwill demonstrated methods used in the biological assay of growth substances and inhibitors, and showed the application of paper chromatography to the separation and identification of growth substances. The test objects used were sections of wheat coleoptiles, unpollinated tomato ovaries, and excised nodes of Coleus. The application of these techniques to the study of problems of fruit set and fruit drop in apples was also illustrated. Another exhibit dealt with the use of $\alpha$-naphthalene acetic acid as a fruit-thinning agent. This substance applied to apple trees within three weeks of petal-fall has been shown to inhibit seed development, thereby leading to an increased drop of developing fruitlets. The method is likely to prove of value for improving fruit size in heavy-cropping varieties and in controlling biennial bearing.

Dr. D. J. D. Nicholas, of the Plant Nutrition Section, had exhibits elucidating the technique of employing Aspergillus niger (Mulder's strain) for the determination of metal micronutrients in soils and plant-tissue homogenates. The assay is specific and quantitative for each micronutrient and gives results which are often below the limits of chemical detection. The trace metal requirements of other species of Aspergillus and Penicillium glaucum were shown to be similar to those of $A$. niger.

Other aspects of the biochemistry of $A$. niger illustrated were sugar utilization, vitamin $\mathbf{B}_{2}$ and $\mathrm{B}_{12}$ production, respiration-rates, amino-acid metabolism and dehydrogenase activity of spores, under various metal deficiencies.

Dr. Nicholas also demonstrated the incidence of iron deficiency in pot-soil culture plants of tomato given nickel, cobalt and zinc, singly and in combination, and in oat given copper only. The metals, including iron, are extracted from fresh leaf tissue with glass-distilled water in micro-mills made of 'Pyrex' glass. Cation exchange resins, for example, 'Zeokarb 215' or 'Amberlites I.R. 100' and '125', are used for concentrating the trace metals and for separating them from the anions. The metal contents of tissue homogenates before and after passage through ion exchange rissins are determined as well as the total amounts in dry matter. The appearance of iron chlorosis is paralleled by a decrease in chloro. phylls $A$ and $B$ as determined by a quartz spectrophotometer.

Another exhibit showed the degree of correlation between final yield of crop plants, grown in factorial experiments, and the mineral status determined for various plant portions, at intervals during the season. In this work, a fractionation of the total mineral nutrients is made by maceration techniques and deep-freeze methods. Correlation coefficients between the results of these methods and those of ash analysis were shown.

In the sand culture investigations under the direction of Dr. E. J. Hewitt, the main work demonstrated comprised a factorial study on cauliflower of the relationships of molybdenum supply to source of nitrogen. In this trial it was shown that leaf symptoms of molybdenum deficiency developed strongly with nitrate or ammonium nitrate but were less marked with nitrite or urea. Ammonium sulphate was toxic alone but not in the presence of added molybdenum-free calcium carbonate. Except for a slight ammonium toxicity, growth was good for all sources of nitrogen in the presence of a normal molybdenum supply.

Molybdenum toxicity (500 p.p.m.) was severe with urea or ammonium sulphate or citrate, but was barely evident with nitrite, nitrate or ammonium nitrate.

Ascorbic acid content of the plants was much reduced by molybdenum deficiency, independent of the type of nitrogen supply. These results suggest a role of molybdenum closely related to nitrogen metabolism but not entirely dependent on the source of nitrogen.

A feature of the work at Long Ashton is the extent of collaboration between the various sections. The Insecticides and Fungicides Section, for example, carries out conjoint work with the Sections of Organic Chemistry, Physical Chemistry, Entomology, Mycology and Pomology. Modifications of the structure of DDT were demonstrated in work by Dr. D. Woodcock, Mr. E. J. Skerrett and Miss P. M. James on the relationship between chemical constitution and insecticidal action. Dr. J. T. Martin's exhibit dealt with the extent and permanence of deposits of DDT and rotenone on apple shoots and on soft fruit. He showed also the progress of investigations on the active principles and methods of assay of derris root.

Dr. W. D. E. Thomas and Mr. S. H. Bennett illustrated the application of a radioantive tracer in following the absorption by apple, chrysanthemum, Coleus and bean plants of a systemic insecticide (bisdimethylaminophosphonous anhydride). The use of the radioanhydride has yielded information on the factors affecting the absorption of the insecticide by leaves, its translocation and its subsequent breakdown. Parallel biological experiments on aphids using the radio-inactive anhydride permit an evaluation of the lethal concentration within the leaves.

Other applications of the use of radioactive tracers were shown in pot culture studies on the phosphate uptake of tomatoes in cobalt-rich soil (Drs. Thomas and Nicholas) and on the effects of clean cultivation compared with grass cover on the phosphate uptake of apple root stocks (Drs. Thomas and C. Bould).

Miss A. Crang and Miss M. Sturdy, of the Domestic Preservation Section, had on view bottled fruit 